\title{
Pro- and anti-inflammatory cytokines in latent autoimmune diabetes in adults, type 1 and type 2 diabetes patients: Action LADA 4
}

\author{
M. N. Pham • M. I. Hawa • C. Pfleger • M. Roden • \\ G. Schernthaner $\cdot$ P. Pozzilli $\cdot$ R. Buzzetti $\cdot$ \\ W. Scherbaum • J. Seissler • H. Kolb • S. Hunter • \\ R. D. G. Leslie • N. C. Schloot • \\ Action LADA Study Group
}

Received: 28 October 2010 /Accepted: 24 January 2011 /Published online: 24 February 2011

(C) Springer-Verlag 2011

\begin{abstract}
Aims/hypothesis Systemic pro- and anti-inflammatory cytokines are associated with both type 1 and type 2 diabetes, while their role in latent autoimmune diabetes in adults (LADA) is unclear. Therefore, we compared cytokine concentrations in patients with LADA, type 1 or type 2 diabetes and healthy individuals to test the hypothesis that differences of cytokine concentrations between all groups are attributable to diabetes type and BMI.
\end{abstract}

M. N. Pham $(\bowtie) \cdot$ C. Pfleger $\cdot$ M. Roden $\cdot$ N. C. Schloot Institute for Clinical Diabetology, German Diabetes Center, Leibniz Center for Diabetes Research

at Heinrich-Heine University Duesseldorf,

Auf'm Hennekamp 65,

40225 Duesseldorf, Germany

e-mail: minh.pham@ddz.uni-duesseldorf.de

M. I. Hawa $\cdot$ R. D. G. Leslie

Blizard Institute of Cell and Molecular Science,

London, UK

C. Pfleger

Benaroya Research Institute,

Seattle, WA, USA

M. Roden • N. C. Schloot

Department of Metabolic Diseases, University of Duesseldorf,

Medical Faculty,

Duesseldorf, Germany

G. Schernthaner

Department of Medicine I, Rudolfstiftung Hospital,

Vienna, Austria

\section{P. Pozzilli}

Department of Endocrinology and Diabetes,

University Campus Bio-Medico,

Rome, Italy
Methods The pro-inflammatory cytokines IL-6 and TNF- $\alpha$, and the anti-inflammatory cytokines IL-1 receptor antagonist (IL-1RA) and IL-10 were measured in 90 participants with type 1 diabetes, 61 with LADA, 465 with type 2 diabetes and 41 control participants using multiple regression models adjusted for BMI, sex, age, blood pressure and diabetes duration.

Results Patients with type 2 diabetes had higher concentrations of systemic IL-1RA, IL- 6 and TNF- $\alpha$ cytokines

R. Buzzetti

Department of Clinical Science, Sapienza University Rome,

Rome, Italy

W. Scherbaum

Department of Endocrinology, Diabetes and Rheumatology,

University of Duesseldorf, Medical Faculty,

Duesseldorf, Germany

J. Seissler

Department of Medicine, Ludwig-Maximillians-University, Munich, Germany

H. Kolb

Department of Immunobiology Research Group,

University of Duesseldorf, Medical Faculty,

Duesseldorf, Germany

S. Hunter

Department of Regional Centre for Endocrinology and Diabetes,

Royal Victoria Hospital,

Belfast, UK 
than patients with either LADA or type 1 diabetes $(p<0.0001$ for all differences). Cytokine concentrations in controls were lower than those in all diabetes types $(p<0.04)$. Increased BMI was positively associated with higher systemic cytokine concentrations in all diabetes types $(p<0.0001)$. Despite the association of cytokines with anthropometric data, differences between diabetes forms persisted also after adjusting analysis for the confounders BMI, age, sex, disease duration and blood pressure $(p<0.04)$.

Conclusions/interpretation Although body mass associates positively with pro- and anti-inflammatory cytokine levels, patients with type 2 diabetes have higher cytokine levels independent of the prevailing BMI. LADA and type 1 diabetes could not be distinguished by systemic cytokines.

Keywords Body mass index Interleukin-6 .

Interleukin-10 · Interleukin-1 receptor antagonist .

Latent autoimmune diabetes in adults . Tumour-necrosis

factor alpha Type 1 diabetes Type 2 diabetes

\section{Abbreviations \\ GADA Glutamic acid decarboxylase antibody \\ IL-1RA IL-1 receptor antagonist \\ LADA Latent autoimmune diabetes in adults}

\section{Introduction}

Type 2 diabetes is characterised by impaired beta cell function and insulin sensitivity and is often accompanied by other metabolic abnormalities [1,2]. These features are accompanied by alterations of the immune system [3-6]. Previous studies found an elevation of systemic pro- and anti-inflammatory cytokine concentrations in patients at risk for diabetes and with overt type 2 diabetes [7, 8]. Obese patients with or without type 2 diabetes also have increased levels of systemic cytokines $[9,10]$. In particular, IL- 6 and TNF- $\alpha$ are produced in abundance in adipose tissue and are thought to contribute to both development of type 2 diabetes and insulin resistance [11-14]. Serum concentrations of IL-10 and IL-1 receptor antagonist (IL-1RA), antiinflammatory cytokines, are also secreted in adipose tissue and are associated with obesity and disease progression of type 2 diabetes $[15,16]$. Treatment with IL-1RA in longterm type 2 diabetes patients has shown to improve $\mathrm{HbA}_{1 \mathrm{c}}$ and to increase endogenous insulin secretion [17].

Type 1 diabetes results from immune-mediated beta cell destruction and is accompanied by islet-directed antibodies, while T cell-mediated and cytokine-mediated cytotoxicity is thought to lead to beta cell destruction $[18,19]$. It has been shown that during insulitis of type 1 diabetes invading immune cells produce cytokines such as IL- $1 \beta$, TNF- $\alpha$ and
IFN- $\gamma$, which are known to be cytotoxic to beta cells [20-26]. After manifestation of type 1 diabetes, T cell reactivity and systemic cytokines such as IL-1RA are associated with endogenous insulin secretion and have been shown to relate to disease progression [27-29].

Latent autoimmune diabetes in adults (LADA) has some clinical features of type 2 diabetes but shows immunological abnormalities similar to those in type 1 diabetes, such as glutamic acid decarboxylase antibody (GADA) [30, 31]. So far it is not understood why disease progression in LADA is slower than in type 1 diabetes despite the immunological similarities. Insulin secretion was reported to be intermediate in LADA compared with type 1 and type 2 diabetes, whereas metabolic syndrome was similar in type 1 diabetes and LADA [32, 33]. The role of pro- and antiinflammatory cytokines in LADA has not yet been investigated.

We tested and compared circulating concentrations of pro-inflammatory cytokines IL- 6 and TNF- $\alpha$, and antiinflammatory cytokines IL-10 and IL-1RA in patients with LADA, type 1 or type 2 diabetes and healthy participants and analysed their associations with body mass, age and sex.

\section{Methods}

The study population consisted of 657 individuals aged from 30 to 70 years, 616 of whom had been diagnosed with diabetes within 5 years before entering this cross-sectional study from the Action LADA cohort. The Action LADA multicentre study was performed to identify immune and clinical risk factors for adult-onset autoimmune diabetes, including its epidemiology, genetic susceptibility, metabolic characteristics and clinical progression [33]. Serum samples were selected on basis of availability and age range. We included 61 individuals with LADA, 90 with type 1 diabetes, 465 with type 2 diabetes and 41 healthy individuals.

Patients with type 1 diabetes were GADA-positive and received insulin treatment after diabetes diagnosis. GADApositive patients aged from 30 to 70 years who did not use insulin treatment for at least 6 months after diagnosis were termed LADA. GADA-negative patients who did not use insulin at least for the first year after diagnosis were defined as having type 2 diabetes. Blood withdrawal from all participants was carried out in the fasting state. The local ethics committee of each study centre approved the study protocol, in accordance with the Declaration of Helsinki. All patients gave written informed consent for the study.

Serum cytokine measurements Serum was collected and stored at $-80^{\circ} \mathrm{C}$ and thawed only once for cytokine analysis. Circulating cytokine concentrations of IL-1RA, IL-6, TNF- $\alpha$ and IL-10 were measured by multiplex-bead 
technology using commercially available kits (Fluorokine MAP; R\&D Systems, Wiesbaden, Germany). The detection limits of the assays were $9.56 \mathrm{pg} / \mathrm{ml}$ for IL-1RA, $0.1 \mathrm{pg} / \mathrm{ml}$ for IL-6, $0.08 \mathrm{pg} / \mathrm{ml}$ for TNF- $\alpha$ and $0.25 \mathrm{pg} / \mathrm{ml}$ for IL-10. For cytokine concentrations lower than the detection limit a value half of the detection limit was assigned (IL-6, $n=46$; IL-1RA, $n=0 ; \mathrm{TNF}-\alpha, n=0)$. Concentration of cytokine IL-10 was only detectable in $44 \%$ of the samples. Immunoassays showed inter-assay variations $<20 \%$ and intra-assay variations $<10 \%$.

Statistical methods Analyses were performed using SAS Enterprise Guide version 4.2 (SAS Institute, Cary, NC, USA) and GraphPad Prism version 4 for Windows (GraphPad Software, La Jolla, California, USA). Continuous variables are presented as medians and interquartile ranges (Q1, 25th percentile; Q3, 75th percentile). First, Gaussian distribution of data was assessed using the KolmogorovSmirnov test. The Kruskal-Wallis and Mann-Whitney $U$ tests were used to compare continuous variables. Fisher's exact test or the $\chi^{2}$ test was performed to evaluate the differences in categorical data with two or more classes. Circulating concentrations of IL-10 were estimated as categorical variables (detectable or not detectable). Data on these analyses were not corrected for multiple comparisons and are therefore descriptive. Univariate correlations between circulating concentrations of cytokines of all groups and BMI, age, sex, blood pressure and diabetes duration were described by Spearman correlation coefficients $(r)$. Associations between tested circulating concentrations of cytokines were carried out with regression analysis adjusted for BMI, age and sex. In addition, we used multivariate regression models to investigate differences of log-transformed cytokine concentrations (dependent variables) in different participant groups adjusted for BMI, age, sex, systolic and diastolic blood pressure and duration of diabetes (independent variables). We tested five models, which adjusted for an increasing number of variables: model 1 , unadjusted; model 2 , sex and age; model 3, age, sex and BMI; model 4, age, sex, BMI and blood pressure; and model 5, age, sex, BMI, blood pressure and diabetes duration. For the analysis of IL-10 logistic regression was performed using the same independent variables as in multiple linear regression models. BMI was calculated as body weight in kilograms divided by the square of the height in meters $\left(\mathrm{kg} / \mathrm{m}^{2}\right)$. For all these descriptive statistical analyses, $p<0.05$ was considered to indicate a statistically significant difference.

\section{Results}

The median age of all patients with diabetes was 54.3 years (Q1: 44.3, Q3: 60.9). Groups differed regarding the median age (Table 1). Patients with type 1 diabetes were younger than those with type 2 diabetes and LADA $(p<0.0001)$. There were no differences in the median ages of the type 1 diabetes and healthy groups or the LADA and type 2 diabetes groups. As expected, the median age at onset in patient groups was different $(p<0.0001)$ : type 1 diabetes patients were younger than type 2 diabetes and LADA patients $(p=0.002$, Table 1$)$. The median duration of diabetes was similar between individuals with type 2, type 1

Table 1 Clinical characteristics of participants with type 1 diabetes, LADA and type 2 diabetes and control individuals

\begin{tabular}{|c|c|c|c|c|c|}
\hline Characteristic & Type 1 diabetes & LADA & Type 2 diabetes & Control & $p$ value \\
\hline$n$ (women/men) & $90(28 / 62)$ & $61(35 / 26)$ & $465(202 / 263)$ & $41(25 / 16)$ & $* *$ \\
\hline Age (years) & $43.2(35.5-53.3)$ & $49.1(39.0-58.5)$ & $56.3(48.1-62.1)$ & $47.7(39.6-53.1)$ & $* * *$ \\
\hline Duration of diabetes (years) & $0.1(0.02-1.6)$ & $0.1(0.04-2.3)$ & $0.1(0.03-1.6)$ & - & NS \\
\hline Age at onset (years) & $44.3(36.9-52.0)$ & $49.0(41.50-58.0)$ & $54.10(46.9-59.0)$ & - & $* * *$ \\
\hline BMI $\left(\mathrm{kg} / \mathrm{m}^{2}\right)$ & $26.1(22.8-29.2)$ & $25.5(23.0-28.8)$ & $30.3(27.0-34.3)$ & $23.5(21.0-27.3)$ & $* * *$ \\
\hline Systolic blood pressure (mmHg) & $120(110-139)$ & $130(119-140)$ & $133(117-145)$ & $112(105-131)$ & $* * *$ \\
\hline Diastolic blood pressure (mmHg) & $80(68-85)$ & $80(71-85)$ & $82(72-89)$ & $80.0(67-81)$ & NS \\
\hline IL-1RA (pg/ml) & $927.3(616.1-1757.0)$ & $942.7(603.4-1261.3)$ & $1167.3(728.6-1972.0)$ & $855.5(570.3-1016.0)$ & $* * *$ \\
\hline IL-6 (pg/ml) & $0.6(0.3-1.4)$ & $0.6(0.3-1.4)$ & $1.1(0.5-2.3)$ & $0.3(0.03-0.5)$ & $* * *$ \\
\hline TNF- $\alpha(\mathrm{pg} / \mathrm{ml})$ & $2.4(1.4-3.4)$ & $2.2(1.4-3.4)$ & $2.9(1.8-4.2)$ & $1.8(1.1-2.5)$ & $* * *$ \\
\hline IL-10 (pg/ml) & $0.01-17.4$ & $0.01-1.9$ & $0.01-49.9$ & $0.1-2.3$ & NS \\
\hline
\end{tabular}

Data are presented as medians and interquartile range (Q1-Q3) if not otherwise indicated

As $56 \%$ of IL-10 serum concentrations were below the detection limit, range (minimum to maximum) is shown

Individual cytokine data are depicted in Fig. 1

$p$ values are derived from comparison of all four groups

$* * p<0.01 ; * * * p<0.001$ 
diabetes and LADA. The median BMI was higher in type 2 diabetes patients than in those with type 1 diabetes or LADA and healthy individuals (all $p<0.0001$ ), while the type 1 diabetes and LADA groups were similar in their median $\operatorname{BMI}(p=0.72)$. The healthy individuals had a normal median BMI.

Comparison of circulating cytokine concentrations in different diabetes types The median circulating concentrations of IL-1RA, IL- 6 and TNF- $\alpha$ were different between all four groups (all $p<0.0001$ ), though we observed an extensive overlap between groups (Fig. 1, Table 1). Type 2 diabetes patients had increased median levels of the antiinflammatory cytokine IL-1RA, and the pro-inflammatory mediators IL- 6 and TNF- $\alpha$ compared with type 1 diabetes patients, those with LADA and healthy participants (all $p<0.03$, Table 2). Compared with healthy participants, type 1 diabetes and LADA patients showed higher median concentrations of IL-1RA, IL-6 and TNF- $\alpha$ (all $p<0.04$, Table 2). Interestingly, group-by-group comparisons revealed no differences in median cytokine concentrations of IL-1RA, IL- 6 and TNF- $\alpha$ between LADA and type 1 diabetes patients (Fig. 1, Table 2). Systemic concentrations of IL-10 showed statistically significant differences between all groups $(p=0.049$, Table 1$)$. In healthy individuals, IL-10 concentrations showed a trend to be lower than in type 1 diabetes ( $p=0.06$, Table 2$)$ and were lower than in patients with LADA $(p=0.003$, Table 2$)$ and type 2 diabetes ( $p=0.007$, Table 2$)$. There were, however, no differences between the diabetes groups, though only $44 \%$ of sera samples had measurable IL- 10 concentrations.

Associations of circulating cytokine concentrations with potential confounders Systemic concentrations of IL-1RA, IL-6 and TNF- $\alpha$ in all groups correlated positively with BMI (IL-1RA, $r=0.35$; IL-6, $r=0.30$; TNF- $\alpha, r=0.20$; all $p<0.0001$; Table 3 ). Circulating concentration of TNF- $\alpha$ in healthy participants did not correlate with BMI after classification of all individuals in groups.

In addition, waist circumference data, as a more representative measure of abdominal obesity, were available for a subgroup ( $n=495,70 \%$ of total cohort) of patients. In this subgroup, a positive correlation between BMI and waist circumference was seen $(r=0.85, p<0.0001)$. Waist circumference also showed a positive correlation with IL-1RA, IL-6 and TNF- $\alpha$ (IL-1RA: $r=0.36$, IL-6: $r=0.33$, TNF- $\alpha: r=0.23$,
Fig. 1 Circulating concentrations of cytokines in control, type 1 diabetes, LADA and type 2 diabetes participants: (a) IL-1RA; (b) IL-10; (c) IL-6; and (d) TNF- $\alpha$. Each point represents the measured cytokine concentrations of an individual. Horizontal lines depict medians. $p$ values of IL-1RA, IL- 6 and TNF- $\alpha$ were calculated with multiple linear regression models (model 1). $p$ values of IL-10 were estimated with logistic regression. ${ }^{*} p<0.05 ; * * p<0.01$; $* * * p<0.001$. T1D, type 1 diabetes; T2D, type 2 diabetes
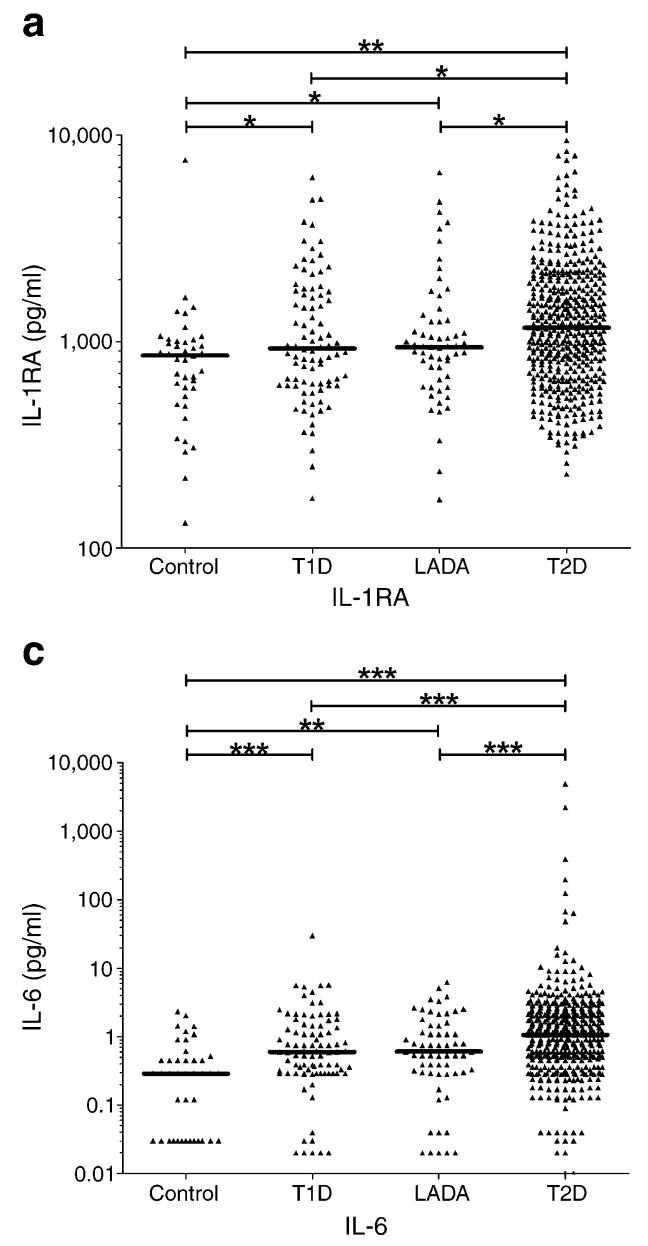

b

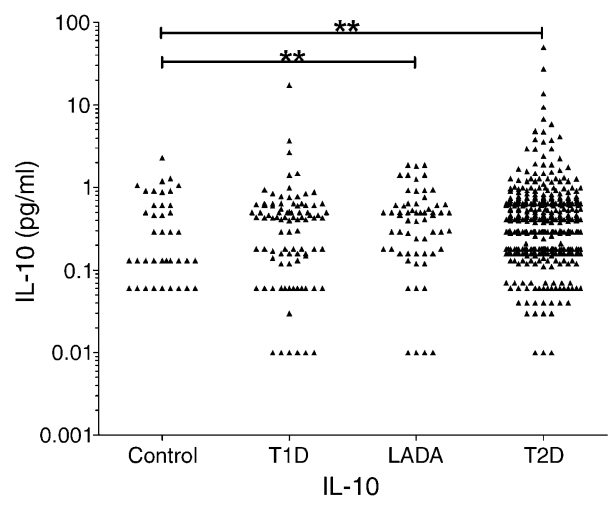

d

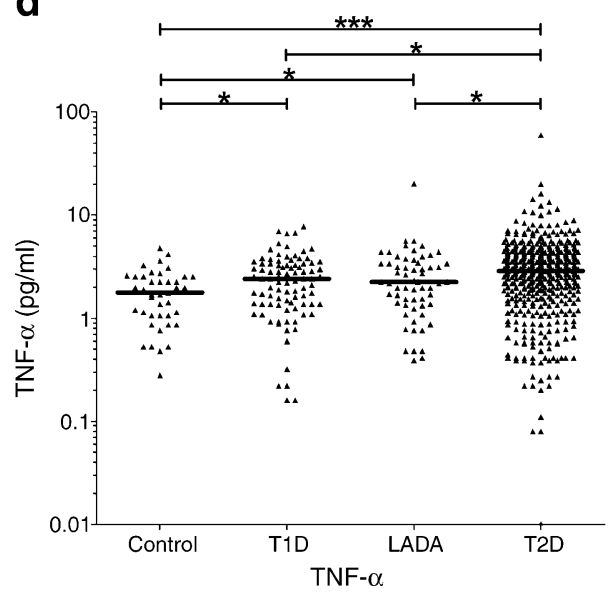


Table 2 Adjusted comparisons between LADA, type 1, type 2 diabetes and control groups

\begin{tabular}{|c|c|c|c|c|c|c|}
\hline $\begin{array}{l}\text { Cytokine/ } \\
\text { model }\end{array}$ & $\begin{array}{l}\text { LADA vs type } 1 \\
\text { diabetes }(\beta)\end{array}$ & $\begin{array}{l}\text { LADA vs type } 2 \\
\text { diabetes }(\beta)\end{array}$ & $\begin{array}{l}\text { Type } 1 \text { diabetes vs } \\
\text { type } 2 \text { diabetes }(\beta)\end{array}$ & $\begin{array}{l}\text { Control vs } \\
\text { LADA }(\beta)\end{array}$ & $\begin{array}{l}\text { Control vs type } 1 \\
\text { diabetes }(\beta)\end{array}$ & $\begin{array}{l}\text { Control vs type } 2 \\
\text { diabetes }(\beta)\end{array}$ \\
\hline \multicolumn{7}{|l|}{ IL-1RA } \\
\hline 1 & 0.06 & $0.17 *$ & $0.04 *$ & $0.02 *$ & $0.05^{*}$ & $0.14 * *$ \\
\hline 2 & 0.06 & $0.17 *$ & $0.03^{*}$ & $0.01^{*}$ & $0.05^{*}$ & $0.16^{* *}$ \\
\hline 3 & 0.05 & $0.18^{*}$ & $0.04 *$ & $0.01^{*}$ & $0.02 *$ & $0.12^{*}$ \\
\hline 4 & 0.06 & $0.33^{* *}$ & $0.01^{*}$ & $0.01^{*}$ & $0.01^{*}$ & $0.21 * * *$ \\
\hline 5 & 0.07 & $0.33 * *$ & $0.01 *$ & - & - & - \\
\hline \multicolumn{7}{|l|}{ IL-6 } \\
\hline 1 & 0.05 & $0.31 * * *$ & $0.26^{* * *}$ & $0.13^{* *}$ & $0.45^{* * *}$ & $0.36 * * *$ \\
\hline 2 & 0.06 & $0.33 * * *$ & $0.21^{*}$ & $0.13^{*}$ & $0.46^{* * *}$ & $0.35^{* * *}$ \\
\hline 3 & 0.04 & $0.23^{*}$ & $0.13^{*}$ & $0.09^{*}$ & $0.38^{* *}$ & $0.27 * * *$ \\
\hline 4 & 0.01 & $0.32 *$ & $0.31^{*}$ & $0.12^{*}$ & $0.41 * *$ & $0.35 * * *$ \\
\hline 5 & 0.02 & $0.31^{*}$ & $0.33^{* *}$ & - & - & - \\
\hline \multicolumn{7}{|l|}{ TNF- $\alpha$} \\
\hline 1 & 0.01 & $0.08^{*}$ & $0.09^{*}$ & $0.04 *$ & $0.11^{*}$ & $0.19 * * *$ \\
\hline 2 & 0.01 & $0.06^{*}$ & $0.05^{*}$ & $0.04^{*}$ & $0.11^{*}$ & $0.09^{*}$ \\
\hline 3 & 0.01 & $0.04 *$ & $0.04 *$ & 0.02 & 0.10 & $0.07 *$ \\
\hline 4 & 0.03 & $0.08^{*}$ & $0.08^{*}$ & 0.01 & 0.01 & $0.06^{*}$ \\
\hline 5 & 0.04 & $0.08 *$ & $0.08^{*}$ & - & - & - \\
\hline \multicolumn{7}{|l|}{ IL-10 } \\
\hline 1 & 0.04 & 0.05 & 0.03 & $0.08 * *$ & 0.16 & $0.09 * *$ \\
\hline 2 & 0.03 & 0.04 & 0.05 & $0.08 * *$ & 0.17 & $0.09 * *$ \\
\hline 3 & 0.04 & 0.04 & 0.06 & $0.08^{* *}$ & 0.16 & $0.09 * *$ \\
\hline 4 & 0.03 & 0.12 & 0.04 & $0.08 * *$ & 0.16 & 0.07 \\
\hline 5 & 0.03 & 0.12 & 0.04 & - & - & - \\
\hline
\end{tabular}

Multiple linear regression models were performed for IL-1RA, IL-6 and TNF- $\alpha$

Cytokines were entered into the models as log-transformed variables; logistic regression analyses were applied for IL-10

Model 1: unadjusted; model 2: age and sex; model 3: age, sex and BMI; model 4: age, sex, BMI and blood pressure (diastolic, systolic); model 5: age, sex, BMI, blood pressure and duration of diabetes

${ }^{*} p<0.05 ; * * p<0.01 ; * * * p<0.001$

all $p<0.0001$ ), similar to the association reported for these cytokines with BMI.

To visualise this effect in all individuals with diabetes we categorised BMI according to the clinical classification of normal weight (18-24.9 kg/m²), overweight (25-29.9 kg/m²), and obesity $\left(\geq 30 \mathrm{~kg} / \mathrm{m}^{2}\right)$ in accordance with WHO definitions. As the number of healthy individuals investigated was low, we display only the results for type 2 diabetes and
Table 3 Correlation between circulating cytokine concentrations and metabolic variables for all groups combined

Correlation analyses between anthropometric variables and IL-1RA, IL-10, TNF- $\alpha$ and IL-6 were performed using Spearman's test ${ }^{*} p<0.05 ; * * p<0.01$; $* * * p<0.001$

\begin{tabular}{lllll}
\hline Variable & IL-1RA $(r)$ & IL-10 $(r)$ & TNF- $\alpha(r)$ & IL-6 $(r)$ \\
\hline Age & -0.003 & -0.03 & $0.18^{* * *}$ & $0.16^{* * *}$ \\
Sex & $0.16^{* * *}$ & 0.07 & -0.03 & 0.02 \\
Diabetes duration & -0.04 & 0.04 & 0.08 & 0.004 \\
Systolic blood pressure & $0.25^{* * *}$ & -0.04 & $0.12^{*}$ & $0.25^{* * *}$ \\
Diastolic blood pressure & $0.17^{* * *}$ & 0.05 & -0.01 & 0.08 \\
BMI & $0.35^{* * *}$ & 0.09 & $0.20^{* * *}$ & $0.30^{* * *}$ \\
Type 1 diabetes & $0.39^{* * *}$ & 0.07 & $0.19^{*}$ & $0.31^{* *}$ \\
LADA & $0.38^{* *}$ & 0.19 & $0.17^{*}$ & $0.23^{* *}$ \\
Type 2 diabetes & $0.31^{* * *}$ & 0.01 & $0.19 * * *$ & $0.29 * * *$ \\
Control & $0.20^{*}$ & 0.26 & 0.18 & $0.45^{* *}$ \\
\hline
\end{tabular}


the merged patient group type 1 diabetes and LADA, named 'autoimmune diabetes', as individuals with type 1 diabetes and LADA were similar in their cytokine level. Participants with type 2 diabetes, as well as those with autoimmune diabetes, showed increased median cytokine concentrations associated with BMI in the case of TNF- $\alpha$, IL-1RA and IL-6 but not for IL-10 (Fig. 2). In both groups, the highest median systemic cytokine concentrations of IL-1RA, IL-6 and TNF$\alpha$ were in individuals with obesity, followed by overweight patients and with the lowest values in patients with normal weight. However, comparison of median IL-6 concentrations between normal weight and overweight individuals in the autoimmune diabetes/type 2 diabetes group did not reveal significant differences (Fig. 2).

In addition, we found higher median concentrations of IL-1RA in women and a positive correlation of serum concentrations of IL-1RA with systolic and diastolic blood pressures (Table 3). Pro-inflammatory cytokines IL-6 and TNF- $\alpha$ were also positively correlated with age and systolic blood pressure. Circulating concentrations of IL-10 did not reveal any correlation with potential confounders (Table 3).

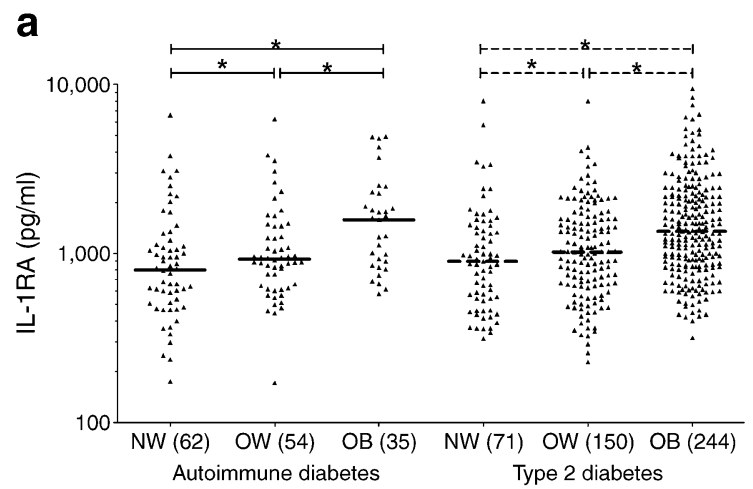

C

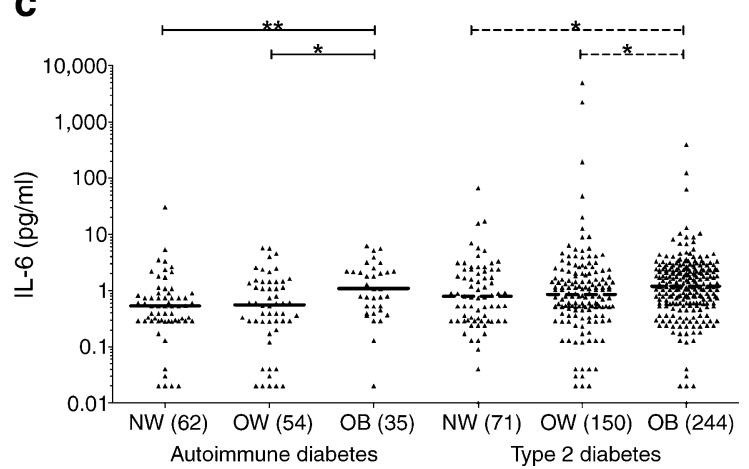

Fig. 2 Circulating cytokine concentrations by BMI of all patients with type 2 diabetes and autoimmune diabetes: (a) IL-1RA; (b) IL-10; (c) IL-6; and (d) TNF- $\alpha$. We pooled type 1 diabetes and LADA participants in one group named 'autoimmune diabetes'. Each point represents the measured cytokine concentrations of an individual. Horizontal lines represent medians. For IL-10 no medians were calculated because too many values were below the detection limit. Normal weight (NW) $18-24.9 \mathrm{~kg} / \mathrm{m}^{2}$; overweight (OW) $25-29.9 \mathrm{~kg} / \mathrm{m}^{2}$; obesity $(\mathrm{OB}) \geq 30 \mathrm{~kg} / \mathrm{m}^{2}$. Number in brackets depicts group size.
Likewise we did not detect an association of waist circumference with IL-10 when analysing the subgroup of $n=495(r=0.04, p=0.28)$.

Associations of circulating concentrations of cytokines Table 4 shows the results of association analysis between cytokines adjusted for BMI, age and sex. All statistically significant associations were positive in all groups with diabetes but not in healthy participants. The strongest associations were between the anti-inflammatory cytokine IL-1RA and pro-inflammatory cytokines IL- 6 and TNF- $\alpha$ (Table 4). Serum concentrations of IL-10 in healthy individuals were positively associated with TNF- $\alpha(\beta=0.7$, $p=0.03$, Table 4 ), but only $44 \%$ of sera had detectable IL- 10 .

Increased concentrations of cytokines in type 2 diabetes after adjustment for confounders As circulating concentrations of cytokines were significantly associated with anthropometric variables we investigated the influence of potential confounding factors on differences in levels of cytokines between the groups. We employed multiple

b

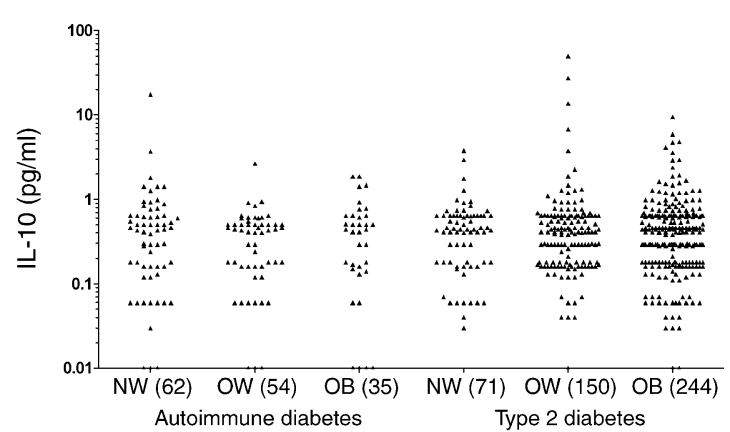

d

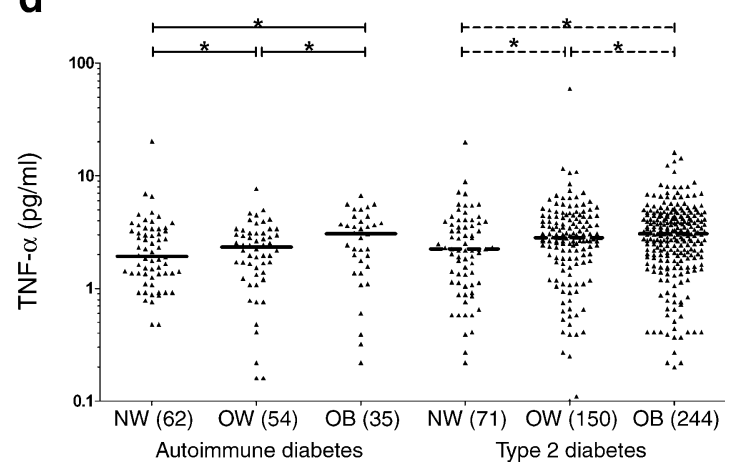

Continuous lines exhibit comparisons of circulating cytokine concentrations between each subgroup with autoimmune diabetes. Broken lines show comparisons of circulating cytokine concentrations between each subgroup with type 2 diabetes. Kruskal-Wallis test resulted in $p<0.0005$ for IL-1RA, IL- 6 and TNF- $\alpha$. Fisher's test for IL-10 gave $p=0.69 . p$ values for IL-1RA, IL-6 and TNF- $\alpha$ were obtained by Mann-Whitney $U$ test: ${ }^{*} p<0.05 ;{ }^{* *} p<0.01$. Additional comparisons between groups revealed further significant $p$ values that are not reported for reasons of clarity 
Table 4 Associations between pro- and anti-inflammatory cytokines adjusted for BMI, age and sex

\begin{tabular}{|c|c|c|c|}
\hline Types/cytokine & IL-1RA $(\beta)$ & IL-6 $(\beta)$ & $\mathrm{TNF}-\alpha(\beta)$ \\
\hline \multicolumn{4}{|l|}{ LADA } \\
\hline IL-1RA & - & & \\
\hline IL-6 & $0.51 * *$ & - & - \\
\hline TNF- $\alpha$ & $0.39 * *$ & $0.54 *$ & -0.20 \\
\hline IL-10 & -0.04 & -0.06 & \\
\hline \multicolumn{4}{|l|}{ Type 1 diabetes } \\
\hline IL-1RA & - & & \\
\hline IL-6 & $0.35^{*}$ & - & - \\
\hline TNF- $\alpha$ & $0.35^{*}$ & $0.15^{* *}$ & -0.08 \\
\hline IL-10 & 0.06 & -0.03 & \\
\hline \multicolumn{4}{|l|}{ Type 2 diabetes } \\
\hline IL-1RA & - & & \\
\hline IL-6 & $0.53 * * *$ & - & - \\
\hline TNF- $\alpha$ & $0.28 * * *$ & $0.86^{* * *}$ & -0.72 \\
\hline IL-10 & 0.03 & -0.001 & \\
\hline \multicolumn{4}{|l|}{ Control } \\
\hline IL-1RA & - & & \\
\hline IL-6 & 0.12 & - & - \\
\hline TNF- $\alpha$ & 0.35 & 0.06 & $0.7 *$ \\
\hline IL-10 & -0.11 & -0.12 & \\
\hline
\end{tabular}

Adjusted association analyses between IL-1RA, IL-6, IL-10 and TNF$\alpha$ were performed with multiple regression analyses

${ }^{*} p<0.05 ;{ }^{* *} p<0.01 ; * * * p<0.001$

regression models to estimate whether differences in IL-1RA, IL-6, TNF- $\alpha$ and IL-10 concentrations between groups, detected in the univariate analysis, persisted after stepwise adjustment for BMI, age, sex, blood pressure and duration of diabetes (Table 2). The similarity of participants with LADA and those with type 1 diabetes and their differences compared with type 2 diabetes patients were maintained after stepwise adjustments. Only the differences for systemic concentrations of TNF- $\alpha$ between healthy control participants vs LADA/type 1 diabetes disappeared after additional adjustments for confounders.

\section{Discussion}

Increased concentrations of systemic cytokines were observed in patients with type 2 diabetes, while levels in patients with LADA and type 1 diabetes were similar. Higher BMI was positively associated with higher systemic cytokine concentrations in all groups. Furthermore, patients with type 2 diabetes exhibited a BMI-independent elevation of systemic cytokines which was not explained by sex, age or blood pressure. We therefore confirmed the hypothesis that increased BMI affects systemic cytokine concentrations in patients with type 2 diabetes and extended these observations to patients with type 1 diabetes and LADA. Similar findings were obtained in healthy control individuals; however, as this group was small and the focus or our investigation was on comparison of type 1 , type 2 diabetes and LADA, further studies are warranted to confirm this finding. Several studies have shown an association between overproduction of pro- and anti-inflammatory cytokines and weight, obesity, adipose tissue and metabolic syndrome $[16,34,35]$. In the present study BMI was used to study obesity, as this marker correlates tightly with waist circumference and both variables are robust risk factors associated with diabetes [36, 37]. In our study, all individuals with higher BMI simultaneously had higher circulating pro- as well as anti-inflammatory cytokine concentrations, regardless of diabetes type. These data point to a positive influence of obesity on the secretion of systemic cytokines independent of diabetes group and it is an additional risk factor for impairment of disease progression in autoimmune diabetes as well as type 2 diabetes.

Our study is the first investigation comparing systemic cytokine concentrations in LADA with type 1 and type 2 diabetes. It has some limitations that should be mentioned. The number of LADA patients compared with type 2 diabetes is relatively low and our definition of LADA as clinical 'type 2 diabetes patients positive for GADA' (and not for other type 1 diabetes related autoantibodies) may be oversimplistic, though similar to that used in previous studies [31-33]. The study design was cross-sectional. A longitudinal follow-up study would be more appropriate and enable the investigation of different stages in progression from healthy control to prediabetic states and overt diabetes. Finally, possible associations between glucose and lipid toxicity, beta cell function and circulating cytokine concentrations in different diabetes groups could not be investigated because suitable data were not collected. As adjustment for the confounding variables age, sex and BMI did not change the associations, it would be of interest to implement measures of glycaemia (fasting glucose, $\mathrm{HbA}_{1 \mathrm{c}}$ ), lipids, insulin secretory capacity and insulin resistance in such patients in the future.

However, in the present study we showed that circulating pro- and anti-inflammatory cytokine concentrations in LADA and type 1 diabetes were similar, but lower compared with type 2 diabetes. This finding is consistent with the proposal that type 1 diabetes and LADA are immunologically similar, occupying different ends of an immunophenotypic spectrum [38]. The present data are in line with our previous observation that the prevalence of metabolic syndrome in type 2 diabetes patients was higher than in patients with LADA or type 1 diabetes [33].

Our current results show that systemic cytokines are positively associated with BMI in addition to diabetes type. 
Previous studies have reported that the elevation of pro- and anti-inflammatory cytokine levels is linked to higher risk of diabetes development [39-41]. We extend these observations as we describe that in addition of BMI, diabetes type is associated with systemic cytokine concentrations. Contrary to our expectation, differences in cytokine concentrations in type 2 diabetes compared with type 1 diabetes and LADA persisted after adjustment for BMI, age, sex, diabetes duration and blood pressure. Overall, these results illustrate that increased circulating concentrations of proand anti-inflammatory cytokines are generally affected by BMI and other potential confounders. This observation did not hold true for IL-10, which was detected in $44 \%$ of individuals only, similar to the findings in previous studies [42-45]. Differences in cytokine concentrations between those with type 2 diabetes and those with both autoimmune diabetes types are not solely explained and influenced by these potential confounders, suggesting a diabetes-type associated immune dysregulation.

Unfortunately, additional variables of the metabolic syndrome according to the IDF definition such as waistto-hip ratio, triacylglycerols, HDL-cholesterol and fasting plasma glucose were not available for this study and we cannot conclude that differences of cytokines between types of diabetes relate, in fact, to disease pathogenesis. Measurement of systemic cytokine concentrations is unlikely to reflect the local inflammatory milieu around disease-related tissue.

Of note, IL-1RA, TNF- $\alpha$ and IL-6 were positively associated in all patients groups, even after adjusting for BMI, age and sex. We speculate that this positive correlation between pro- and anti-inflammatory cytokines may reflect a counter-regulatory attempt to ameliorate inflammation in patients with diabetes, associated with a general upregulation of immune responses. Interestingly, the Whitehall II study on IL-1RA also reported an upregulation of both pro- and anti-inflammatory mediators 6 years before the diagnosis of type 2 diabetes [7, 8].

In conclusion, we confirmed that greater systemic cytokine concentrations associate with body mass in type 2 diabetes. Further, systemic cytokines were similarly lower in both type 1 diabetes and LADA, but higher than in control participants. Thus, factors other than cytokines are responsible for the difference of the clinical phenotype between type 1 diabetes and LADA.

Acknowledgements This study was performed with samples obtained from Action LADA Group, which was supported by the EU (contract number No: QLG1-CT-2002-01886). Financial support for these analyses came from German Diabetes Foundation 'Das zuckerkranke Kind'. This study was supported in part by a grant from the German Federal Ministry of Education and Research (BMBF) to the German Center for Diabetes Research (DZD e.V.). We thank C. Herder and A. Strom of Institute for Clinical Diabetology at German Diabetes Center for their advice.
Duality of interest The authors declare that there is no duality of interest associated with this manuscript.

\section{References}

1. Butler AE, Janson J, Bonner-Weir, Ritzel R, Rizza RA, Butler PC (2003) Beta-cell deficit and increased beta-cell apoptosis in humans with type 2 diabetes. Diabetes 52:102-110

2. Hotamisligil GS (2006) Inflammation and metabolic disorders. Nature 444:860-867

3. Shoelson SE, Lee J, Goldfine AB (2006) Inflammation and insulin resistance. J Clin Invest 116:1973-1801

4. Pickup JC, Crook MA (1998) Is type 2 diabetes mellitus a disease of the innate immune system? Diabetologia 41:1241-1248

5. Pickup JC, Mattock MB, Chusney GD, Burt D (1997) NIDDM as a disease of the immune system: association of acute phase reactants and interleukin-6 with metabolic syndrome. Diabetologia 40:1286-1292

6. Kolb H, Mandrup-Poulsen T (2005) An immune origin of type 2 diabetes? Diabetologia 48:1038-1050

7. Herder C, Brunner EJ, Rathmann W et al (2009) Elevated levels of the anti-inflammatory interleukin-1 receptor antagonist precede the onset of type 2 diabetes: the Whitehall II study. Diabetes Care $32: 421-423$

8. Carstensen M, Herder C, Kivimäki M et al (2010) Accelerated increase in serum interleukin-1 receptor antagonist (IL-1Ra) starts 6 years before diagnosis of type 2 diabetes: whitehall II prospective cohort study. Diabetes 59:1222-1227

9. Herder C, Zierer A, Koenig W, Roden M, Meisinger C, Thorand B (2009) Transforming growth factor-beta 1 and incident type 2 diabetes: results from the MONICA/KORA case cohort study, 1984-2002. Diabetes Care 32:1921-1923

10. He L, He M, Lv X, Pu D, Su P, Liu Z (2010) NF-kappaB binding activity and pro-inflammatory cytokines expression correlate with body mass index but not glycosylated haemoglobin in Chinese population. Diab Res Clin Pract 90:73-80

11. Ventre J, Doebber T, Wu M et al (1997) Targeted disruption of the tumor necrosis factor-alpha gene: metabolic consequences in obese and nonobese mice. Diabetes 46:1526-1531

12. Hotamisligil GS, Arner P, Caro JF, Atkinson RL, Spiegelman BM (1995) Increased adipose tissue expression of tumor necrosis factor-alpha in human obesity and insulin resistance. J Clin Invest 95:2409-2415

13. Ziccardi P, Nappo F, Giugliano G et al (2002) Reduction of inflammatory cytokine concentrations and improvement of endothelial functions in obese women after weight loss over one year. Circulation 105:804-809

14. Vozarova B, Weyer C, Hanson K, Tataranni PA, Bogardus C, Pratley RE (2001) Circulating interleukin-6 relation to adiposity, insulin action, and insulin secretion. Obes Res 97:414-417

15. Juge-Aubry CE, Somm E, Pernin A et al (2005) Adipose tissue is a regulated source of interleukin-10. Cytokine 29:270-274

16. Juge-Aubry CE, Somm E, Giusti V et al (2003) Adipose tissue is a major source of interleukin-1 receptor antagonist: upregulation in obesity and inflammation. Diabetes 52:1104-1110

17. Larsen CM, Faulenbach M, Vaag A et al (2007) Interleukin-1receptor antagonist in type 2 diabetes mellitus. N Engl J Med 356:1517-1526

18. Atkinson MA, Eisenbarth GS (2001) Type 1 diabetes: new perspectives on disease pathogenesis and treatment. Lancet 358:221-229

19. Tisch R, McDevitt H (1996) Insulin-dependent diabetes mellitus. Cell 85:291-297 
20. Cnop M, Welsh N, Jonas JC, Jörns A, Lenzen S, Eizirik DL (2005) Mechanisms of pancreatic beta-cell death in type 1 and type 2 diabetes: many differences, few similarities. Diabetes 54 (supp12):S97-S107

21. Eizirik DL, Colli ML, Ortis F (2009) The role of inflammation in insulitis and beta-cell loss in type 1 diabetes. Nat Rev Endocrinol 5:219-226

22. Mandrup-Poulsen T (1990) Cytokine and free radicals as effector molecules in the destruction of pancreatic beta cells. Curr Top Microbiol Immunol 164:169-193

23. Campbell IL, Oxbrow L, Harrison LC (1991) Reduction in Insulitis following administration of IFN-gamma and TNF-alpha in the NOD mouse. J Autoimm 4:249-262

24. Nicoletti F, Zaccone P, Di Marco R et al (1998) Paradoxical antidiabetogenic effect of gamma-interferon in DP-BB rats. Diabetes 47:32-38

25. Ortis F, Naamane N, Flamez D et al (2010) Cytokines interleukin$1 \beta$ and tumor necrosis factor- $\alpha$ regulate different transcriptional and alternative splicing networks in primary $\beta$-cells. Diabetes 59:358-374

26. Hoorens A, Stangé G, Pavlovic D, Pipeleers D (2001) Distinction between interleukin-1-induced necrosis and apoptosis of islet cells. Diabetes 50:551-557

27. Pfleger C, Meierhoff G, Kolb H, Schloot NC, p520/521 Study Group (2010) Association of $\mathrm{T}$ cell reactivity with beta-cell function in recent onset type 1 diabetes patients. J Autoimmun 34:127-135

28. Pfleger C, Kaas A, Hansen L et al (2008) Relation of circulating concentrations of chemokine receptor CCR5 ligands to C-peptide, proinsulin and $\mathrm{HbAlc}$ and disease progression in type 1 diabetes. Clin Immunol 128:57-65

29. Pfleger C, Mortensen HB, Hansen L et al (2008) Association of IL-1ra and adiponectin with C-peptide and remission in patients with type 1 diabetes. Diabetes 57:929-937

30. Leslie RD, Kolb H, Schloot NC et al (2008) Diabetes classification: grey zones, sound and smoke: Action LADA 1. Diab Metab Res Rev 24:511-519

31. Hosszúfalusi N, Vatay A, Rajczy K et al (2003) Similar genetic features and different islet cell autoantibody pattern of latent autoimmune diabetes (LADA) compared with adult-onset type 1 diabetes with rapid progression. Diabetes Care 26:452-457

32. Gottsäter A, Landin-Olsson M, Fernlund P, Lernmark A, Sundkvist G (1993) Beta-cell function in relation to islet cell antibodies during the first $3 \mathrm{yr}$ after clinical diagnosis in type 2 diabetic patients. Diabetes Care 16:902-910
33. Hawa MI, Thivolet C, Mauricio D et al (2009) Metabolic syndrome and autoimmune diabetes: Action LADA 3. Diabetes Care 32:160-164

34. Meier CA, Bobbioni E, Gabay C, Assimacopoulos-Jeannet F, Golay A, Dayer JM (2002) IL-1 receptor antagonist serum levels are increased in human obesity: a possible link to the resistance to leptin. J Clin Endocrinol Metab 87:1184-1188

35. Dandona P, Weinstock R, Thusu K, Abdel-Rahman E, Aljada A, Wadden T (1998) Tumor necrosis factor-alpha in sera of obese patients: fall with weight loss. J Clin Endocrinol Metab 83:29072910

36. Shoelson SE, Herrero L, Naaz A (2007) Obesity, inflammation, and insulin resistance. Gastroenterology 132:2169-2180

37. Shoelson SE, Goldfine AB (2009) Getting away from glucose: fanning the flames of obesity-induced inflammation. Nat Med 15:373-374

38. Rolandsson O, Palmer JP (2010) Latent autoimmune diabetes in adults (LADA) is dead: long live autoimmune diabetes. Diabetologia 53:1250-1253

39. Pradhan AD, Manson JAE, Rifai N, Buring JE, Ridker (2001) Creactive Protein, Interleukin-6, and risk of developing type 2 diabetes mellitus. JAMA 286:327-334

40. Devaraj S, Glaser N, Griffen S, Wang-Polagruto J, Miguelino E, Jialal I (2006) Increased monocytic activity and biomarkers of inflammation in patients with type 1 diabetes. Diabetes 55:774-779

41. Basu S, Larsson A, Vessby J, Vessby B, Berne C (2005) Type 1 diabetes is associated with increased cyclooxygenase and cytokine-mediated inflammation. Diab Care 28:1371-1375

42. Trøseid M, Seljeflot I, Hjerkinn EM, Arnesen H (2009) Interleukin-18 is a strong predictor of cardiovascular events in elderly men with the metabolic syndrome: synergistic effect of inflammation and hyperglycemia. Diabetes Care 32:486-492

43. Hartkamp A, Geenen R, Bijl M, Kruize AA, Godaert GL, Derksen RH (2004) Serum cytokine levels related to multiple dimensions of fatigue in patients with primary Sjogren's syndrome. Ann Rheum Dis 63:1335-1337

44. Mysliwiec M, Zorena K, Balcerska A, Mysliwska J, Lipowski P, Raczynska K (2006) The activity of N-acetyl-beta-D-glucosaminidase and tumour necrosis factor-alpha at early stage of diabetic retinopathy development in type 1 diabetes mellitus children. Clin Biochem 39:851-856

45. Weihrauch MR, Manzke O, Beyer M et al (2005) Elevated serum levels of CC thymus and activation-related chemokine (TARC) in primary Hodgkin's disease potential for a prognostic factor. Cancer Res 65:5516-5519 